Check for updates

Cite this: Phys. Chem. Chem. Phys.,

2021, 23, 4311

\section{Flexible to rigid: IR spectroscopic investigation of a rhenium-tricarbonyl-complex at a buried interface $\uparrow$}

\author{
Kerstin T. Oppelt, (D) * Laurent Sevéry, (D) $\neq$ Mirjam Utters, (iD S. David Tilley (iD and \\ Peter Hamm (i)
}

\begin{abstract}
This work explores the solid-liquid interface of a rhenium-tricarbonyl complex embedded in a layer of zirconium oxide deposited by atomic layer deposition (ALD). Time-resolved and steady state infrared spectroscopy were applied to reveal the correlations between the thickness of the ALD layer and the spectroscopic response of the system. We observed a transition of the molecular environment from flexible to rigid, as well as limitations to ligand exchange and excited state quenching on the embedded complexes, when the ALD layer is roughly of the same height as the molecules.
\end{abstract}

\section{Introduction}

Inspired by research on the conversion of light energy to chemical energy ${ }^{1}$ and heterogeneous photochemical water reduction systems, which are often based on immobilized molecular photosensitizers and catalysts, ${ }^{2,3}$ this work aims to investigate the solid/liquid interface of these types of systems. They are often comprised of transition metal complexes as photoactive and/or catalytic compounds, which are bound to (transition) metal oxide scaffolds. ${ }^{4-8}$ Additional stabilizing layers, deposited by ALD or other methods, ${ }^{9-12}$ have been explored to protect adsorbed molecules against desorption by limiting solvent exposure of the binding site. These layers are usually very thin compared to the height of the molecules. We consider the situation where a cover layer eventually tops the molecules to be an interesting point to investigate.

ALD is a self-saturating and self-limiting vapour coating technique, which is based on chemical reactions between a precursor and the functional groups of the surface that is coated. ${ }^{13,14}$ It offers atomic level control of layer thickness and layer composition and furthermore, it enables conformal coating of complex 3D surface structures. ALD can be applied to stabilize photoelectrodes against corrosion, and has been used on top of sensitizer- and/or catalyst-functionalized semiconductor surfaces in dye-sensitized solar cells and photoelectrochemical cells. ${ }^{15,16}$ As such, ALD seems to be the best method to control the

Department of Chemistry, University of Zurich, Winterthurerstr. 190, CH-8057 Zürich, Switzerland. E-mail: kerstin.oppelt@chem.uzh.ch; Tel: +41 446354477

$\dagger$ Electronic supplementary information (ESI) available. See DOI: 10.1039/ d0cp06546c

\# Present address: Department of Chemistry, University of California, Berkeley, California 94720, USA. thickness and nature of the deposited cover layer described previously. ${ }^{13,14,17}$

There are quite a few studies about the influence of deposited ALD layers on the interface between a metal oxide semiconductor and an adsorbed molecule. Parameters that have been tested are not only long term stability, ${ }^{12}$ but also charge transfer to/from the metal oxide, as well as energy transfer between adsorbed molecules. ${ }^{2,18}$ Most studies were performed using either $\mathrm{TiO}_{2}$ or $\mathrm{Al}_{2} \mathrm{O}_{3}$ ALD cover layers. Here, we aim to investigate the interface between the liquid phase and a transition metal complex anchored to mesoporous $\mathrm{ZrO}_{2}$ with $\mathrm{ZrO}_{2}$ cover layers. $\mathrm{ZrO}_{2}$ is a commonly employed coating material and has the advantage of being transparent to the light from near infrared to the visible region due to its band gap of about $5.4 \mathrm{eV} \cdot{ }^{19}$ Furthermore, $\mathrm{ZrO}_{2}$ is quite inert, and, therefore, it is expected to only barely influence any chemical reactions. ${ }^{20,21}$

We apply FTIR, transient Vis-pump-IR-probe (tr-IR), as well as two-dimensional infrared (2D-IR) spectroscopy. We will show that each of these methods can cover special aspects of the molecular behaviour at this interface. The ground state reactivity and accessibility can be observed by ligand exchange experiments, while transient Vis-pump-IR probe spectroscopy can cover many aspects of photochemical reactivity. Solvation dynamics can be investigated by two-dimensional infrared spectroscopy. With increasing coverage the response of the system should transition from rather flexible to very rigid at a cover layer thickness that corresponds to the height of the molecules on the surface. The thickness of one ALD cycle is about $1 \AA{ }^{22,23}$ while the height of the molecule is $\approx 9 \AA$, as estimated from a published crystal structure of a similar complex. ${ }^{24}$ Thus, we expect the transition to happen with 10 ALD cycles, and furthermore, that the transition will be relatively abrupt. 


\section{Materials and methods}

\subsection{Sample}

We investigate the complex $\operatorname{Re}\left(2,2^{\prime}\right.$-bipyridyl-4,4'-bisphosphonic acid)(CO) ${ }_{3} \mathrm{Cl}$ (Fig. 1, ReP-Cl) ${ }^{20,24,25}$ anchored on a $200 \mathrm{~nm}$ thick mesoporous layer consisting of $\mathrm{ZrO}_{2}$ nanoparticles $(20-40 \mathrm{~nm}$ diameter) on $\mathrm{CaF}_{2}$ substrate. Synthesis of the rhenium complex was performed according to published procedures. ${ }^{20,24,26}$

The mesoporous film was prepared by spin coating of diluted nanoparticle solutions $(1.1 \mathrm{~g}$ Solaronix Z-SP and $5 \mathrm{ml}$ EtOH) on UV grade $\mathrm{CaF}_{2}$ windows (Crystran) as substrates. After sintering the nanoporous layers at $550{ }^{\circ} \mathrm{C}$ on a hot plate, the substrates (with the sintered films) were immersed in ethanolic solutions of the rhenium complex $(0.2 \mathrm{mM})$ for at least $12 \mathrm{~h}$ to ensure a good coverage. Samples were then rinsed with copious amounts of ethanol and then dried with a stream of nitrogen. FITR spectra were measured with a Bruker Tensor 27 FTIR spectrometer. The IR absorbance and the film thickness derived from scanning electron microscopy imaging was used to confirm that the surface coverage is on average a monolayer of ReP-Cl. Details can be found in the ESI. $\dagger$

ALD was performed using a Picosun R-200 ALD reactor using TEMAZ (tetrakis-(ethylmethylamino)-zirconium(Iv), Sigma-Aldrich, $>99.99 \%$ ) as the zirconium source, Milli-Q water as oxygen source, and $\mathrm{N}_{2}$ as carrier gas for $\mathrm{ZrO}_{2}$. TEMAZ was kept at $110{ }^{\circ} \mathrm{C}$ during the deposition using a heating mantle, while the $\mathrm{H}_{2} \mathrm{O}$ was kept at $25{ }^{\circ} \mathrm{C}$ using a Peltier cooler. The substrate temperature was held at $150{ }^{\circ} \mathrm{C}$ during the entire deposition process. For flat surfaces, a precursor pulse consisted of an opening of the precursor source to the main chamber $(2.0 \mathrm{~s}$ for TEMAZ, $0.1 \mathrm{~s}$ for $\mathrm{H}_{2} \mathrm{O}$ ), followed by a $20 \mathrm{~s}$ purge with $\mathrm{N}_{2}$. To deposit conformal layers on mesoporous substrates, a stopped flow method was used to increase the residence time of the precursor molecules in the reactor. To determine the thickness of the grown ALD layers, a small piece of silicon wafer was inserted along with the samples into the reactor. The growth rate per cycle ( $\approx 1 \AA$ ) was consistent with the literature ${ }^{22}$ and was determined by ellipsometry of the wafer piece before and after deposition using a J. A. Woollam Alpha-SE ellipsometer. The deposited layer was modelled using the WVase software.

The mesoporous film used here is relatively thin compared to previously studied samples, ${ }^{20}$ leading to a small absorption cross section of the adsorbed molecules. Thicker mesoporous films turned out to be only incompletely covered by the ALD layer, and most likely only at their very surface. In addition to using a thinner mesoporous layer, we adjusted pulsing times of reagent and water vapour during the ALD process to ensure that

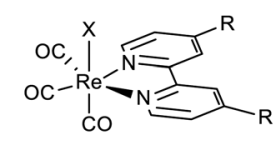

ReP-Cl R= $\mathrm{PO}_{3} \mathrm{H}_{2} \mathrm{X}=\mathrm{Cl}$ ReP-NCS R=PO $\mathrm{H}_{2} \mathrm{X}=\mathrm{NCS}$

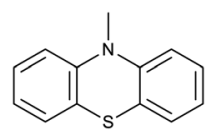

MePTZ
Fig. 1 Chemical structures of the rhenium complex and the electron donor addressed in this work.
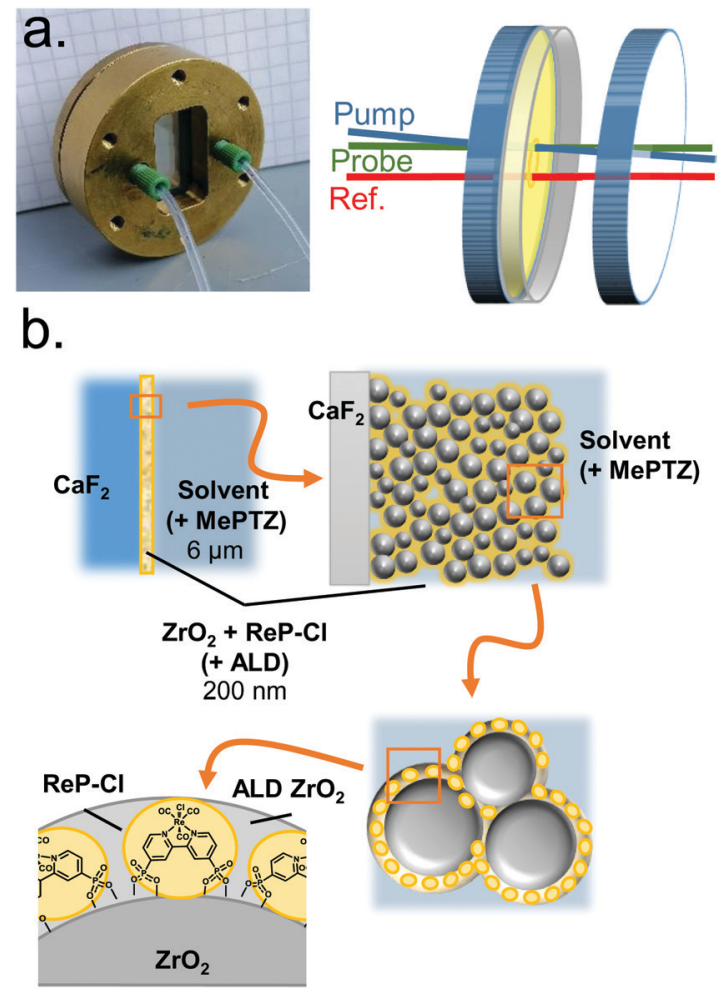

Fig. 2 Schematics of the samples. (a) Image of the measurement cell and sketch of the sample between $\mathrm{CaF}_{2}$ windows and liquid layer, together with the laser beams in the applied transmission geometry. (b) Schematic views and magnifications of the substrate, the structure of the mesoporous film as well as the adsorbed $\mathrm{ReP}-\mathrm{Cl}$ molecule and the deposited $\mathrm{ZrO}_{2}$ ALD layer.

both can diffuse through the whole cross section of the mesoporous film. Furthermore, it is important to deposit the ALD layers at a temperature low enough to avoid changes of the immobilized molecules on the surface and thus to their functionality. ${ }^{14,27}$

Fig. 2 schematically shows the sample geometry, and the assembly used in the time resolved measurements described below.

\subsection{Time-resolved spectroscopy}

The laser system used to measure the transient IR spectroscopy consisted of two synchronized Ti:Sa lasers operating at $2.5 \mathrm{kHz}$. This spectrometer setup allowed for pump-probe delays from approximately $10 \mathrm{ps}$ (caused by the jitter of the electronic synchronization) to $40 \mu \mathrm{s}$. Frequency doubling of the first laser created pump pulses centred around $400 \mathrm{~nm}$. The second laser generated tunable mid-IR probe pulses in a home-built optical parametric amplifier (OPA). ${ }^{28}$

The pump laser energy was $60-80 \mathrm{~nJ} \mathrm{~cm}{ }^{-2}$, and the duration was stretched to $4 \mathrm{ps}$. This low excitation density in combination with purging of either the pure solvent or the quencher solution with argon ensured mild conditions during the experiments, and avoided triplet-triplet annihilation that we observed at higher excitation densities. ${ }^{20}$ The sample spot was constantly changed during the measurement using a home-build 2D raster scanner 
with magnetic linear motors to avoid sample damage by constant focus on only one spot. Furthermore, solvent and quencher solutions were constantly exchanged in the sample cell using a peristaltic pump. The sample cell consisted of the $\mathrm{CaF}_{2}$ window with the prepared oxide layer and a clean $\mathrm{CaF}_{2}$ window separated by a $6 \mu \mathrm{m}$ MYLAR spacer.

After dispersing the probe pulse in a spectrograph right after the sample, it was detected by a $2 \times 64$ pixel MCT detector, which covered a $200 \mathrm{~cm}^{-1}$ spectral window with a resolution of about $4 \mathrm{~cm}^{-1}$. For data analysis, wavenumbers were corrected using the previously recorded FTIR spectra and the data were normalized by the ground state bleach minimum around $2035 \mathrm{~cm}^{-1}$ at delay zero.

2D-IR measurements were performed with a $1 \mathrm{kHz}$ Ti:Sa laser system, as described elsewhere. ${ }^{29}$ A different spectrograph/detector combination was used, leading to a spectral resolution of $2 \mathrm{~cm}^{-1}$.

\section{Results}

We explored different IR spectroscopic methods to probe the degree to which ReP-Cl was covered by the ALD layer: FTIR, transient Vis-pump-IR-probe and 2D-IR spectroscopy. FTIR was complemented by chemical exchange reactions. In either case, we used the carbonyl stretch modes of the rhenium complex as observables, which are known to be very sensitive to changes in the molecule environment, ${ }^{30-32}$ the oxidation state of the Re center, and the ligand system. ${ }^{33-35}$ The three tricarbonyl signals around $2040 \mathrm{~cm}^{-1}, 1950 \mathrm{~cm}^{-1}$ and $1900 \mathrm{~cm}^{-1}$ are assigned to the $\mathrm{a}^{\prime}(1)$ mode (symmetric stretch vibration of all COs), the $a^{\prime \prime}$ (antisymmetric stretching of equatorial COs) and the $\mathrm{a}^{\prime}(2)$ mode (antisymmetric stretching of axial $\mathrm{CO}$ and equatorial COs), respectively. ${ }^{36}$

\subsection{FTIR spectroscopy}

In a first step, we monitored the influence of the ALD cover layer on the adsorbed molecules by FTIR spectroscopy. Fig. 3 shows FTIR spectra of dry samples of ReP-Cl on $\mathrm{ZrO}_{2}$ without cover layer (red line) and covered with 10, 20 or 30 cycles of $\mathrm{ZrO}_{2}$ ALD (blue, yellow and green lines). The spectra significantly change upon deposition of ALD layers, reflecting a different environment around the CO groups of ReP-Cl. The largest frequency shift and broadening of the spectra happens between 0 and 10 cycles, and the trend gradually continues beyond. The two different blue and yellow spectra were taken from independently prepared samples, evidencing the reproducibility of the preparation process.

The observed trend is comparable to the results by Song et al. ${ }^{37}$ where a similar Re compound on mesoporous $\mathrm{TiO}_{2}$ has been covered with ALD layers of $\mathrm{Al}_{2} \mathrm{O}_{3}$. They also observed a change in the vibrational frequency, spectral line width and oscillator strength of the carbonyl modes. They explained this behaviour by less clustering between adjacent rhenium molecules, referring to the work of Laaser et $a .^{38}$

\subsection{Ligand exchange}

In a second step, we performed exchange experiments of the axial chloride with thiocyanate $\left(\mathrm{SCN}^{-}\right)$, similar to previously

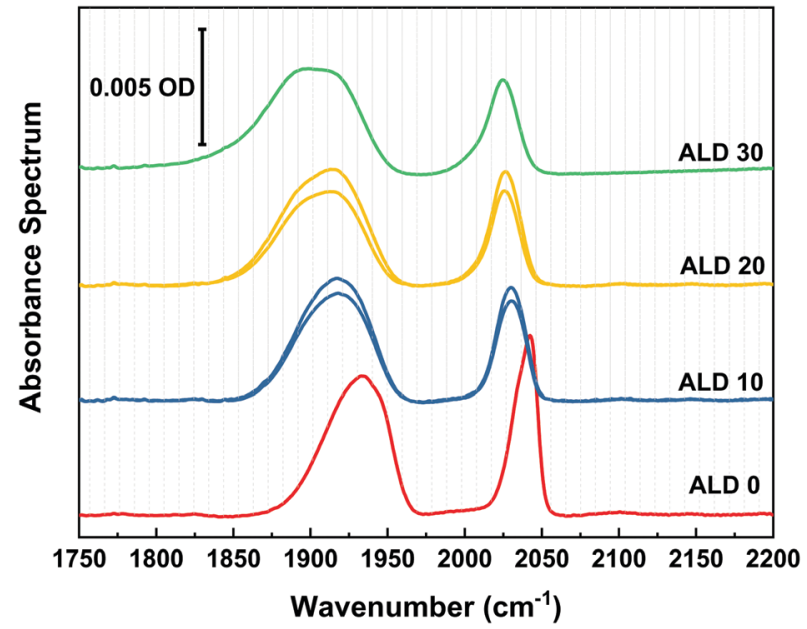

Fig. 3 FTIR spectra of dry samples of $\mathrm{ReP}-\mathrm{Cl}$ on $\mathrm{ZrO}_{2}$ without cover layer (red line) and covered with 10, 20 or 30 cycles of $\mathrm{ZrO}_{2}$ ALD (blue, yellow and green lines, respectively). The two different blue and yellow spectra were taken from independently prepared samples.

published exchange of $\mathrm{Cl}^{-}$on $\mathrm{Ru}$ and $\mathrm{Ir}$ complexes with $\mathrm{CN}^{-} .^{27}$ To that end, the samples were kept in a $100 \mathrm{mM}$ KSCN solution in methanol for 16 days, and rinsed with methanol afterwards. The final product in this reaction is the isothiocyanate $\left(\mathrm{NCS}^{-}\right){ }^{39-41}$ Fig. 4 (dashed lines) exhibits a $\mathrm{NCS}^{-}$stretching band (the C-N stretching mode of the isothiocyanate ligand) at 0 and 10 ALD cycles, but not for thicker ALD layers. In addition, frequency shifts are observed, which are expected upon ligand exchange but hard to disentangle from the environment effect shown in Fig. 3. We hypothesize that for preventing $\mathrm{a} \mathrm{Cl}^{-}$ligand loss, we would at least have to cover the molecule up to its height on the surface $(\approx 9 \AA)$. The height was estimated from a published crystal structure of a similar complex. ${ }^{24}$ Accordingly,

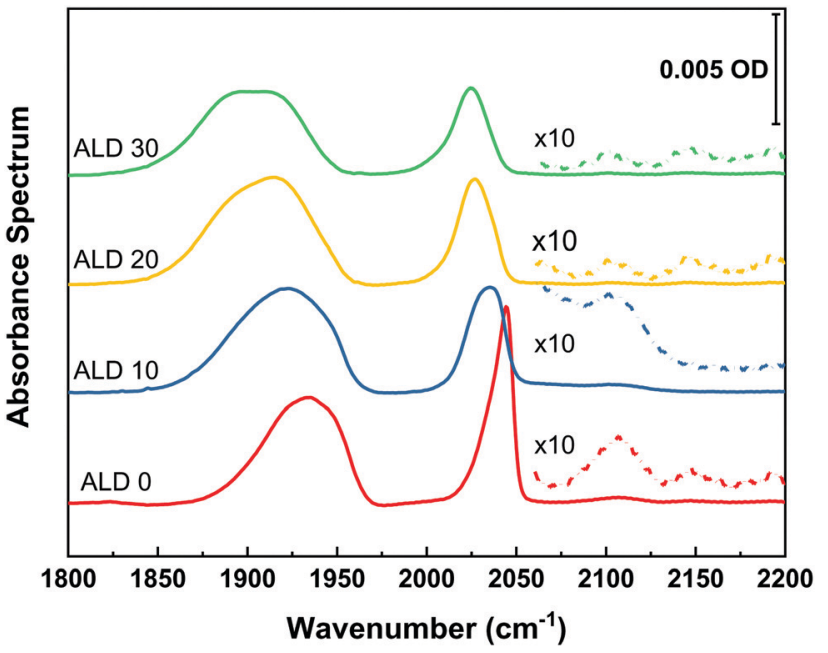

Fig. 4 FTIR spectra of the Re complex with different numbers of ALD cycles after storage of the samples in a KSCN solution in $\mathrm{MeOH}$ to test $\mathrm{Cl}^{-}$ ligand exchange for $\mathrm{NCS}^{-}$. The dash lines show the spectral region of the $\mathrm{NCS}^{-}$stretching vibration with 10 -fold magnification. 
ligand exchange is still possible at 10 ALD layers, albeit weakly and no longer occurs for any of the thicker layers.

\subsection{Transient IR spectroscopy}

The next set of experiments determined up to how many ALD layers the photoexcited complex remains accessible for charge transfer from an electron donor in solution. We utilize this as a measure for how deep the complex is embedded in the ALD film. After photoexcitation, the complex undergoes ultrafast intersystem crossing. The resulting triplet excited state is best seen at delay times $<10$ ns in Fig. 5 by the red shift of the $\mathrm{a}^{\prime}(1)$ mode with a signal appearing at $2060 \mathrm{~cm}^{-1}$. This excited state can be reductively quenched by an electron donor from solution, ${ }^{42,43}$ in our case $50 \mathrm{mM} N$-methyl phenothiazine (MePTZ) in ethanol, which is a reversible electron donor. Electron transfer to the Re complex is usually observed by a marker mode occurring at $1860 \mathrm{~cm}^{-1}$. This band is clearly seen in the case of the sample without ALD layer, see Fig. 5a, red box. It appears at about $10 \mathrm{~ns}$, and disappears again on a $\mu \mathrm{s}$ timescale due to recombination with oxidized electron donor

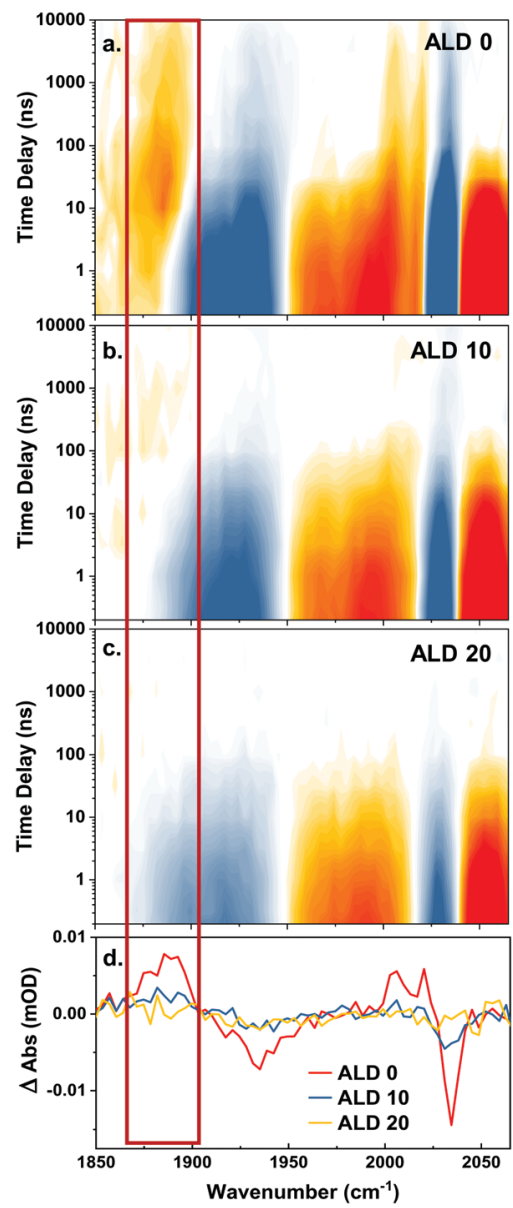

Fig. 5 Transient IR spectroscopy of a sample (a) without ALD layer, (b) with 10 ALD cycles, and (c) with 20 ALD cycles. All data have been measured in presence of $50 \mathrm{mM} \mathrm{N}$-methyl-phenothiazine as quencher. The red box marks the region where the $\mathrm{Re}^{-}$marker mode is observed. Panel (d) shows transient cuts at $1 \mu \mathrm{s}$, with the marker mode indicated as well. molecules. ${ }^{20}$ The same spectral signature can also be discerned for the sample with 10 ALD cycles (Fig. 5b), albeit much weaker. For thicker layers (Fig. 5c), reductive quenching of the Re complex can no longer be observed. Fig. 5d highlights this behaviour by showing transient cuts at $1 \mu \mathrm{s}$.

Besides the $\mathrm{a}^{\prime}(2)$ and the $\mathrm{a}^{\prime \prime}$ bands of the reduced ReP-Cl (the signal marked with the red box in Fig. 5a-d) there is also a parallel shift of the $\mathrm{a}^{\prime}(1)$ to lower energy (positive signal around $2010 \mathrm{~cm}^{-1}$ at $t>100 \mathrm{~ns}$ ) in Fig. 5a and b. Another clear sign for the presence/absence of the photo reaction is given by persisting ground state bleach signals $(t>100 \mathrm{~ns})$, depicted in blue in Fig. $5 \mathrm{a}-\mathrm{c}$ from $1870 \mathrm{~cm}^{-1}$ to $1950 \mathrm{~cm}^{-1}\left(\mathrm{a}^{\prime}(2)\right.$ and $\left.\mathrm{a}^{\prime \prime}\right)$ and especially at $2040 \mathrm{~cm}^{-1}\left(a^{\prime}(1)\right)$. This negative signal persists longer than the acquisition window $(10 \mu \mathrm{s})$. Similarly to the previously described marker mode it can still be observed for the case of 10 ALD layers, but not any more for 20 layers (red and blue vs. yellow lines in Fig. 5d).

\subsection{D-IR spectroscopy}

2D-IR spectroscopy measures the solute-solvent interactions of the CO modes of ReP-Cl with its environment, as well as the timescales on which they are modulated by a process called spectral diffusion. ${ }^{30,32,44-46}$ That is, 2D-IR spectroscopy correlates the vibrational frequency upon excitation with that upon probing at a later time. In a highly fluctuating environment (like a solvent exposed surface), this correlation is quickly lost, while we expect a longer memory in a rigid ALD matrix.

The 2D-IR spectra of the uncovered ReP-Cl surface immersed in ethanol are shown in the top row of Fig. 6 . The blue signal is the ground state bleach, the red signal the excited state absorption. Shortly after excitation (left spectrum), both are elongated along the diagonal of the $2 \mathrm{D}$ plot, reflecting complete memory of the excitation frequency. After $30 \mathrm{ps}$, this correlation is lost and the signal has a mostly horizontal orientation. Thus, the probe frequency has become independent of the excitation frequency. The opposite case, a sample covered with 15 layers, is shown in the bottom row of Fig. 6. An inhomogeneously elongated lineshape can also be seen at $500 \mathrm{fs}$. However,

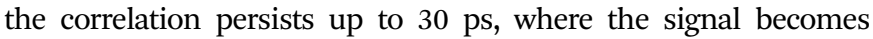
smaller due to excited state relaxation but hardly changes its shape. As often observed, the intensity of the ground state contribution on the diagonal is larger than that of the excited state absorption, and hence remains more distinct after decay. The middle pair of spectra in Fig. 6 highlights the intermediate case with 10 ALD layers, where the bands are inhomogeneously broadened at early times (500 fs), as in the other two cases. At $30 \mathrm{ps}$, the situation is in between that for the uncovered sample and that with 15 ALD cycles, indicating partial, but not complete, loss of correlation.

\section{Discussion and conclusion}

We presented four complementary IR spectroscopy methods to discern to what extent ReP-Cl is covered by an ALD cover layer. Two of these methods, simple FTIR spectroscopy (Fig. 3) as well as 2D-IR spectroscopy (Fig. 6), measure the interactions of 


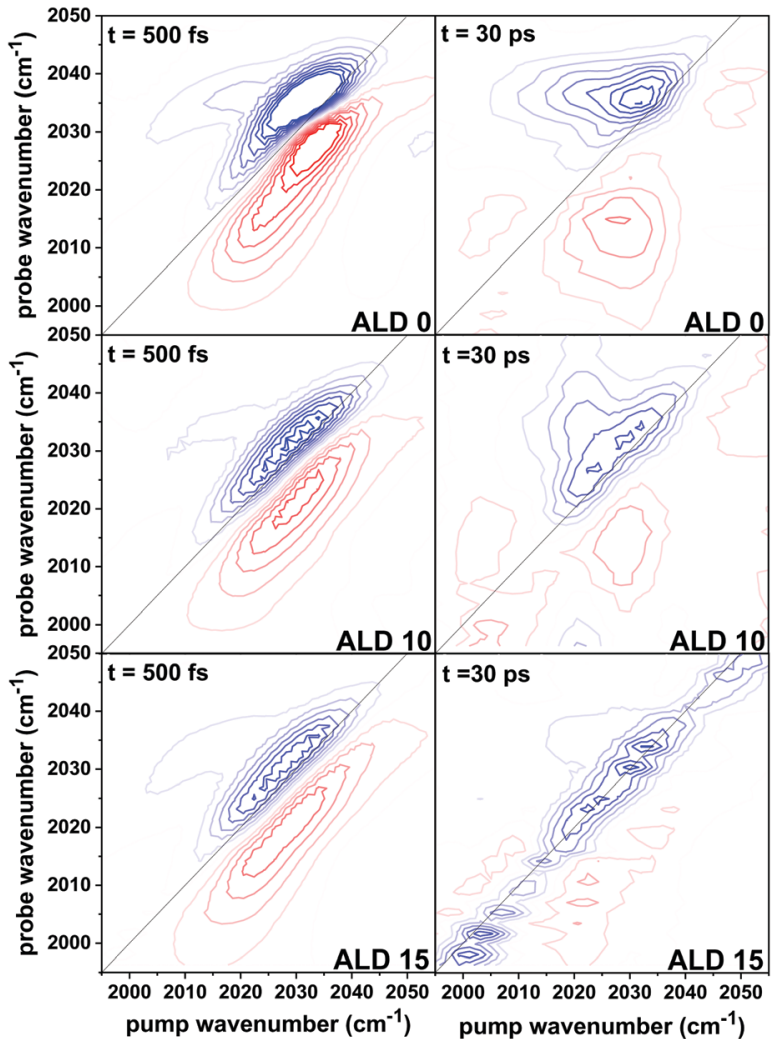

Fig. 6 2D-IR results of the $a^{\prime}(1)$ band of $\mathrm{ReP}-\mathrm{Cl}$ on $\mathrm{ZrO}_{2}$ at 500 fs and 30 ps waiting time for different thicknesses of the $\mathrm{ZrO}_{2}$ ALD cover layer: 0 , 10 and 15 cycles.

the - $\mathrm{CO}$ modes of ReP-Cl with their immediate environment, i.e., the solvent and/or the $\mathrm{ZrO}_{2}$ matrix. The other two methods, ligand exchange (Fig. 4) and reductive quenching by an electron donor (Fig. 5), measure to what extent the immobilized molecules are still accessible by molecules in solution.

All methods consistently show that the transition between solvent exposed and fully covered happens at 10 ALD layers, as anticipated from the height of each ALD layer and the size of the molecule. However, the extent to which the transition is complete at 10 ALD layers varies when comparing the different methods. For example, ligand exchange with $\mathrm{NCS}^{-}$seems to be possible with 10 ALD layers to almost the same extent as without any cover (Fig. 4, compare height of the -NCS bands shown as red and blue dashed lines), while reductive quenching by MePTZ is barely happening (Fig. 5d, compare height of the $\mathrm{Re}^{-}$ band shown as red and blue lines). This can probably be explained by the much smaller size of the $\mathrm{SCN}^{-}$ions in comparison to the electron donor molecule MePTZ. The size of the latter is about the same as that of the bipyridyl ligand of ReP-Cl. Thus, in the present case, it is not possible to inhibit ligand exchange (stabilization of the whole molecule), while retaining charge transfer to and from the complex (in order to use it as a photosensitizer).

Also spectral diffusion mostly stops at 10 ALD cycles already, indicating that the - $\mathrm{CO}$ modes are either in direct contact with the completely static $\mathrm{ZrO}_{2}$ matrix, or with solvent molecules whose flexibility is severely reduced, since they themselves are
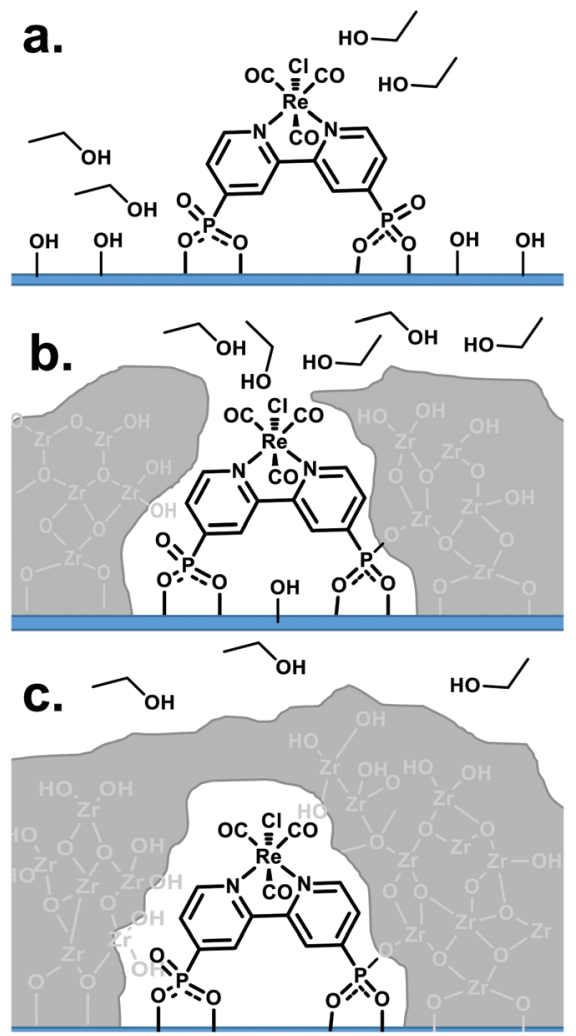

Fig. 7 Explanation for the transition of the ReP-Cl system from a semiflexible to a very rigid system due to increasing coverage of the molecule by solid amorphous $\mathrm{ZrO}_{2}$ : panel (a) shows the uncovered sample, (b) the transition happening at 10 ALD cycles, and (c) the situation $\mathrm{ReP}-\mathrm{Cl}$ is completely covered at $\gtrsim 20$ ALD cycles.

in direct contact with the $\mathrm{ZrO}_{2}$ matrix. This observation is very similar to published work of water in small reverse micelles. In these confined spaces (the water pool is around $2 \mathrm{~nm}$ in diameter) water molecules behave very differently from bulk water. Spectral diffusion slows down and the vibrational lifetime increases as well. The dynamics are dominated by interfacial water molecules that are in contact with the head group of the surfactant forming the reverse micelles. ${ }^{47,48}$ In combination with the results from ligand exchange (Fig. 4), we conclude that with 10 ALD layers, access to ReP-Cl is possible only via relatively small pores.

In conclusion, Fig. 7 shows how we envision the structural properties of the ALD overlayer at the transition when ReP-Cl just gets covered. Access for other molecules to the $\mathrm{ReP}-\mathrm{Cl}$ is strongly reduced (Fig. 7a and b) and the flexibility of the ReP-Cl molecule also decreases dramatically within this rigid cavity that is formed by a surrounding $\mathrm{ZrO}_{2}$ layer. This situation is reached once the ALD layer just exceeds the height of ReP-Cl and after that (Fig. 7c) the observable changes are small.

\section{Conflicts of interest}

There are no conflicts to declare. 


\section{Acknowledgements}

We gratefully acknowledge financial support by the Swiss National Science Foundation (Grant CRSII2_160801/1) and the University Research Priority Program (URPP) for Solar Light to Chemical Energy Conversion (LightChEC) of the University of Zurich. Imaging was performed with support of the Center for Microscopy and Image Analysis, University of Zurich.

\section{Notes and references}

1 D. G. Nocera, Acc. Chem. Res., 2017, 50, 616-619.

2 H. Tian, ChemSusChem, 2015, 8, 3746-3759.

3 B. Zhang and L. Sun, Chem. Soc. Rev., 2019, 48, 2216-2264.

4 R. E. Bangle, M. J. Mortelliti, L. Troian-Gautier, J. L. Dempsey and G. J. Meyer, J. Phys. Chem. C, 2020, 124, 25148-25159.

5 P. Xu and T. E. Mallouk, J. Phys. Chem. C, 2019, 123, 299-305.

6 K. L. Orchard, D. Hojo, K. P. Sokol, M.-J. Chan, N. Asao, T. Adschiri and E. Reisner, Chem. Commun, 2017, 53, 12638-12641.

7 J. Willkomm, N. M. Muresan and E. Reisner, Chem. Sci., 2015, 6, 2727-2736.

8 M. A. Gross, A. Reynal, J. R. Durrant and E. Reisner, J. Am. Chem. Soc., 2014, 136, 356-366.

9 J. Guo, C. She and T. Lian, J. Phys. Chem. C, 2007, 111, 8979-8987. 10 K. Hanson, M. D. Losego, B. Kalanyan, D. L. Ashford, G. N. Parsons and T. J. Meyer, Chem. Mater., 2013, 25, 3-5. 11 M. D. Losego and K. Hanson, Nano Energy, 2013, 2, 1067-1069. 12 A. K. Vannucci, L. Alibabaei, M. D. Losego, J. J. Concepcion, B. Kalanyan, G. N. Parsons and T. J. Meyer, Proc. Natl. Acad. Sci. U. S. A., 2013, 110, 20918-20922.

13 C. Detavernier, J. Dendooven, S. Pulinthanathu Sree, K. F. Ludwig and J. A. Martens, Chem. Soc. Rev., 2011, 40, 5242-5253.

14 K. Kanomata, K. Tokoro, T. Imai, P. Pansila, M. Miura, B. Ahmmad, S. Kubota, K. Hirahara and F. Hirose, Appl. Surf. Sci., 2016, 497-502.

15 D. Wang, M. V. Sheridan, B. Shan, B. H. Farnum, S. L. Marquard, B. D. Sherman, M. S. Eberhart, A. Nayak, C. J. Dares, A. K. Das, R. M. Bullock and T. J. Meyer, J. Am. Chem. Soc., 2017, 139, 14518-14525.

16 M. D. Brady, L. Troian-Gautier, T. C. Motley, M. D. Turlington and G. J. Meyer, ACS Appl. Mater. Interfaces, 2019, 11, 27453-27463.

17 V. Miikkulainen, M. Leskelä, M. Ritala and R. L. Puurunen, J. Appl. Phys., 2013, 113, 021301.

18 H. J. Son, C. H. Kim, D. W. Kim, N. C. Jeong, C. Prasittichai, L. Luo, J. Wu, O. K. Farha, M. R. Wasielewski and J. T. Hupp, ACS Appl. Mater. Interfaces, 2015, 7, 5150-5159.

19 C. Prasittichai, J. R. Avila, O. K. Farha and J. T. Hupp, J. Am. Chem. Soc., 2013, 135, 16328-16331.

20 K. Oppelt, R. Fernández-Terán, R. Pfister and P. Hamm, J. Phys. Chem. C, 2019, 123, 19952-19961.

21 K. Oppelt, M. Mosberger, J. Ruf, R. Fernández-Terán, B. Probst, R. Alberto and P. Hamm, J. Phys. Chem. C, 2020, 124, 12502-12511.

22 D. M. Hausmann, E. Kim, J. Becker and R. G. Gordon, Chem. Mater., 2002, 14, 4350-4358.
23 X. Wang, S. K. Ghosh, M. Afshar-Mohajer, H. Zhou, Y. Liu, X. Han, J. Cai, M. Zou and X. Meng, J. Mater. Res., 2020, 35, 804-812.

24 C. D. Windle, E. Pastor, A. Reynal, A. C. Whitwood, Y. Vaynzof, J. R. Durrant, R. N. Perutz and E. Reisner, Chem. - Eur. J., 2015, 21, 3746-3754.

25 K. Neuthe, F. Bittner, F. Stiemke, B. Ziem, J. Du, M. Zellner, M. Wark, T. Schubert and R. Haag, Dyes Pigm., 2014, 104, 24-33.

26 J. M. Smieja and C. P. Kubiak, Inorg. Chem., 2010, 49, 9283-9289.

27 L. Sévery, S. Siol and S. Tilley, Inorganics, 2018, 6, 105.

28 P. Hamm, R. A. Kaindl and J. Stenger, Opt. Lett., 2000, 25, 1798-1800.

29 J. Helbing and P. Hamm, J. Opt. Soc. Am. B, 2011, 28, 171.

30 L. M. Kiefer, J. T. King and K. J. Kubarych, Acc. Chem. Res., 2015, 48, 1123-1130.

31 L. M. Kiefer and K. J. Kubarych, J. Phys. Chem. A, 2015, 119, 959-965.

32 L. M. Kiefer and K. J. Kubarych, Coord. Chem. Rev., 2018, 372, 153-178.

33 A. Cannizzo, A. M. Blanco-Rodríguez, A. El Nahhas, J. Šebera, S. Záliš, A. Vlček and M. Chergui, J. Am. Chem. Soc., 2008, 130, 8967-8974.

34 B. Probst, A. Rodenberg, M. Guttentag, P. Hamm and R. Alberto, Inorg. Chem., 2010, 49, 6453-6460.

35 C. She, N. A. Anderson, J. Guo, F. Liu, W. H. Goh, D. T. Chen, D. L. Mohler, Z. Q. Tian, J. T. Hupp and T. Lian, J. Phys. Chem. B, 2005, 109, 19345-19355.

36 J. B. Asbury, Y. Wang and T. Lian, Bull. Chem. Soc. Jpn., 2002, 75, 973-983.

37 J. Song, A. Ge, B. Piercy, M. D. Losego and T. Lian, Chem. Phys., 2018, 512, 68-74.

38 J. E. Laaser, J. R. Christianson, T. A. Oudenhoven, Y. Joo, P. Gopalan, J. R. Schmidt and M. T. Zanni, J. Phys. Chem. C, 2014, 118, 5854-5861.

39 A. M. Blanco Rodríguez, A. Gabrielsson, M. Motevalli, P. Matousek, M. Towrie, J. Šebera, S. Záliš and A. Vlček, J. Phys. Chem. A, 2005, 109, 5016-5025.

$40 \mathrm{H}$. Takeda, K. Koike, H. Inoue and O. Ishitani, J. Am. Chem. Soc., 2008, 130, 2023-2031.

41 M. Mosberger, B. Probst, B. Spingler and R. Alberto, Eur. J. Inorg. Chem., 2019, 3518-3525.

42 M. Guttentag, A. Rodenberg, R. Kopelent, B. Probst, C. Buchwalder, M. Brandstätter, P. Hamm and R. Alberto, Eur. J. Inorg. Chem., 2012, 59-64.

43 J. Hawecker, J.-M. Lehn and R. Ziessel, J. Chem. Soc., Chem. Commun., 1984, 6, 328-330.

44 S. T. Roberts, J. J. Loparo and A. Tokmakoff, J. Chem. Phys., 2006, 125, 084502.

45 J. P. Kraack, D. Lotti and P. Hamm, J. Chem. Phys., 2015, 142, 212413.

46 M. T. Zanni, Proc. Natl. Acad. Sci. U. S. A., 2016, 113, 4890-4891. 47 E. E. Fenn, D. B. Wong, C. H. Giammanco and M. D. Fayer, J. Phys. Chem. B, 2011, 115, 11658-11670.

48 R. Costard, N. E. Levinger, E. T. J. Nibbering and T. Elsaesser, J. Phys. Chem. B, 2012, 116, 5752-5759. 